\title{
Mutations and karyotype in myelodysplastic syndromes: TP53 clusters with monosomal karyotype, RUNX1 with trisomy 21, and SF3B1 with inv(3) (q21q26.2) and del(11q)
}

\author{
Ayalew Tefferi ${ }^{1}$, Dame Idossa ${ }^{1}$, Terra L. Lasho ${ }^{1}$, Mythri Mudireddy ${ }^{1}$, Christy Finke', Sahrish Shah ${ }^{1}$, Maura Nicolosi ${ }^{1}$, \\ Mrinal M. Patnaik $\mathbb{B}^{1}$, Animesh Pardanani ${ }^{1}$, Naseema Gangat ${ }^{1}$, Curt A. Hanson ${ }^{2}$ and Rhett P. Ketterling ${ }^{3}$
}

\begin{abstract}
Next-generation sequencing (NGS) studies have now established the presence of sometimes multiple somatic mutations in the majority of patients with myelodysplastic syndromes (MDS) ${ }^{1,2}$. Some of these mutations, including ASXL1, TP53, RUNX1, EZH2, and SRSF2, have been shown to adversely affect overall or leukemia-free survival, independent of each other and conventional risk models ${ }^{2}$. More recent studies have further suggested associations of certain mutations in MDS with specific cytogenetic abnormalities. In this regard, one study employed NGS in 22 MDS patients with $\operatorname{der}(1 ; 7)(\mathrm{q} 10 ; \mathrm{p} 10)$ and 32 with $-7 / \operatorname{del}(7 q)^{3}$; the most frequently mutated genes in the former were RUNX1 (41\%), ASXL1 (23\%), $E Z H 2$ (18\%), and DNMT3A (18\%) and in the latter TP53 (28\%), ASXL1 28\%, SETBP1 (22\%), and TET2 (19\%). Accordingly, the authors suggested an association between $\operatorname{der}(1 ; 7)(\mathrm{q} 10 ; \mathrm{p} 10)$ and RUNX1 mutations ${ }^{3}$. In another NGS study of 43 patients with del(5q)-associated MDS, recurrent mutations among 22 patients with del(5q) syndrome included ASXL1 (14\%), TET2 (14\%), SF3B1 (9\%), TP53 (5\%), RUNX1 (5\%), DNMT3A (5\%), and WT1 $(5 \%)^{4}$; more advanced cases displayed higher frequency of TP53 mutations. Such observations carry both pathogenetic and practical relevance, especially in deciphering the prognostic interaction between mutations and karyotype.
\end{abstract}

\footnotetext{
Correspondence: Ayalew Tefferi (tefferi.ayalew@mayo.edu)

'Division of Hematology, Departments of Internal and Laboratory Medicine, Mayo Clinic, Rochester, MN, USA

${ }^{2}$ Division of Hematopathology, Departments of Internal and Laboratory

Medicine, Mayo Clinic, Rochester, MN, USA

Full list of author information is available at the end of the article
}

In a recent communication, we reported on 179 MDS patients in whom information was available for NGSderived mutational status, and showed an adverse overall and leukemia-free survival impact from ASXL1, SETBP1, or TP53 mutations/variants and SRSF2, IDH2, CSF3R mutations/variants, respectively ${ }^{2}$. The prognostic contribution of these mutations was independent of coexisting mutations, number of mutations, age, and, for the large part, the revised international prognostic scoring system (IPSS-R) ${ }^{2}$; an apparent association between SF3B1 mutations and favorable prognosis was no longer evident after analysis was adjusted for IPSS-R. In the current study, we sought to discover specific associations between mutations and karyotype and clarify the interindependent prognostic contribution of mutations vs. karyotype.

The study population $(N=179)$ consisted of patients with primary MDS who were informative for both karyotype and NGS data. The diagnosis of MDS and leukemic transformation was according to the 2008 World Health Organization (WHO) criteria ${ }^{5}$. Cytogenetic analysis and reporting was done according to the International System for Human Cytogenetic Nomenclature (ISCN) criteria $^{6}$. Cytogenetic reports were re-reviewed and grouped into categories that are found to be informative, on preliminary analysis of associations with specific mutations. A 27-gene panel NGS study was performed on bone marrow DNA specimens, and queried genes included TET2, DNMT3A, IDH1, IDH2, ASXL1, EZH2, SUZ12, SRSF2, SF3B1, ZRSR2, U2AF1, PTPN11, TP53, SH2B3, RUNX1, CBL, NRAS, JAK2, CSF3R, FLT3, 
KIT, CALR, MPL, NPM1, CEBPA, IKZF1, and SETBP1. Altered DNA sequences were deemed as mutations or variants, if they were associated with a hematologic malignancy (as identified by COSMIC database), or if they have not been associated with a dbSNP. All statistical analyses considered clinical and laboratory parameters obtained at the time of mutation screening.

Clinical and laboratory features of the 179 study patients included median age 73 years (range 28-96), males 68\%, median hemoglobin $10 \mathrm{~g} / \mathrm{dl}$ (range 6.9-14.8), median leukocyte count $3.6 \times 10^{9} / 1$ (range 0.8-20), median platelet count $91 \times 10^{9} / 1$ (range 4-599). Risk distribution according to IPSS-R was very high $11 \%$, high $18 \%$, intermediate $17 \%$, low $38 \%$, and very low $16 \%$. Abnormal karyotype was reported in 107 (60\%) patients with the most frequent being normal karyotype $(n=78$; $43.6 \%)$, monosomal karyotype $(n=22 ; 12.3 \%)$, sole +8 $(n=14 ; 7.8 \%)$, sole $\operatorname{del}(5 \mathrm{q})(n=13 ; 7.3 \%)$, sole $-7 /$ del (7q) $(n=6 ; 3.4 \%)$, sole del(11q) $(n=5 ; 2.8 \%)$, sole +21 $(n=4 ; 2.2 \%)$, sole trisomies other than +8 and $+21(n=$ $5 ; 2.8 \%)$, sole del(20q) $(n=4 ; 2.2 \%)$, sole inv(3)(q21q26.2) $(n=2 ; 1.1 \%)$; complex non-monosomal $(n=3 ; 1.7 \%)$, der $(1 ; 7)(\mathrm{q} 10 ; \mathrm{p} 10)(n=3 ; 1.7 \%)$, and other sole or double abnormalities $(n=20 ; 11.2 \%)$. At least one mutation/ variant was seen in 147 (82\%) patients; 58 (32\%) patients harbored one, 48 (27\%) two, and 41 (23\%) three or more. Mutations/variants detected included ASXL1 $(n=53$; $30 \%)$, TET2 $(n=44 ; 25 \%), S F 3 B 1(n=36 ; 20 \%), U 2 A F 1$ $(n=28 ; 16 \%), \operatorname{SRSF2}(n=28 ; 16 \%), \operatorname{TP} 53(n=23 ; 13 \%)$, RUNX1 ( $n=19 ; 11 \%)$, DNMT3A ( $n=18 ; 10 \%), I D H 2$ $(n=11 ; 6 \%), E Z H 2 \quad(n=7 ; 4 \%), C E B P A \quad(n=6 ; 3 \%)$, SETBP1 $(n=5 ; 3 \%), \operatorname{IDH} 1(n=5 ; 3 \%), \operatorname{CSF} 3 R(n=5 ; 3 \%)$, $\operatorname{KIT}(n=3 ; 2 \%), C B L(n=2 ; 1 \%), J A K 2(n=2 ; 1 \%), C A L R$ $(n=1 ; 0.5 \%)$, and FLT3 $(n=1 ; 0.5 \%)$.

Table 1 lists mutations which showed significant associations with specific cytogenetic categories. The most notable associations were between monosomal karyotype and TP53 mutations $(p<0.0001$; mutational frequency of $82 \%$ vs. $<10 \%$ in all other abnormal cytogenetic categories), RUNX1 and $+21 \quad(p<0.0001$; mutational frequency of $100 \%$ vs. $<35 \%$ in all other abnormal cytogenetic categories), and SF3B1 and $\operatorname{del}(11 \mathrm{q})$ and inv (3)(q21q26.2) ( $p=0.0001$; mutational frequency of $80 \%$ and $100 \%$, respectively, vs. $<20 \%$ in all other abnormal cytogenetic categories). Patients with trisomy 21 also frequently harbored ASXL1 (75\%) and SRSF2 (75\%) mutations. Other associations included $A S X L 1$ with +8 , SRSF2 with other sole trisomies, $-7 / \operatorname{del}(7 \mathrm{q})$ with $I D H 1$ and U2AF1 mutations. The close association between TP53 mutations and monosomal karyotype was not further modified by the presence or absence of monosomy 17 , as part of their monosomal karyotype; 7 (32\%) of the 22 cases with monosomal karyotype harbored monosomy 17. Similarly, the specific type of RUNX1 mutation did not

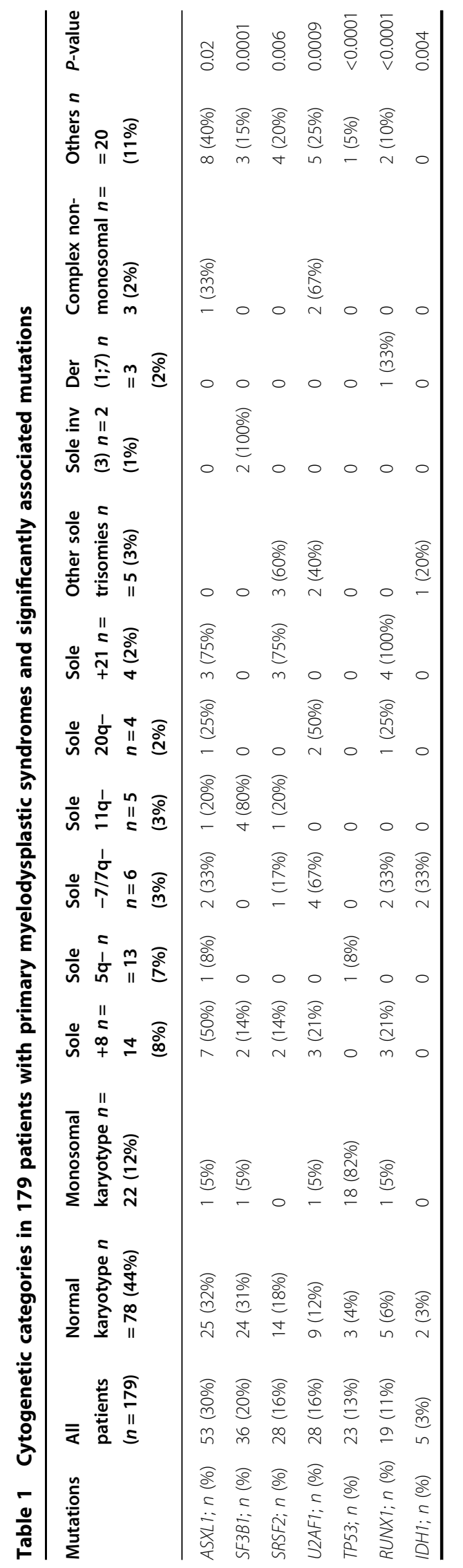




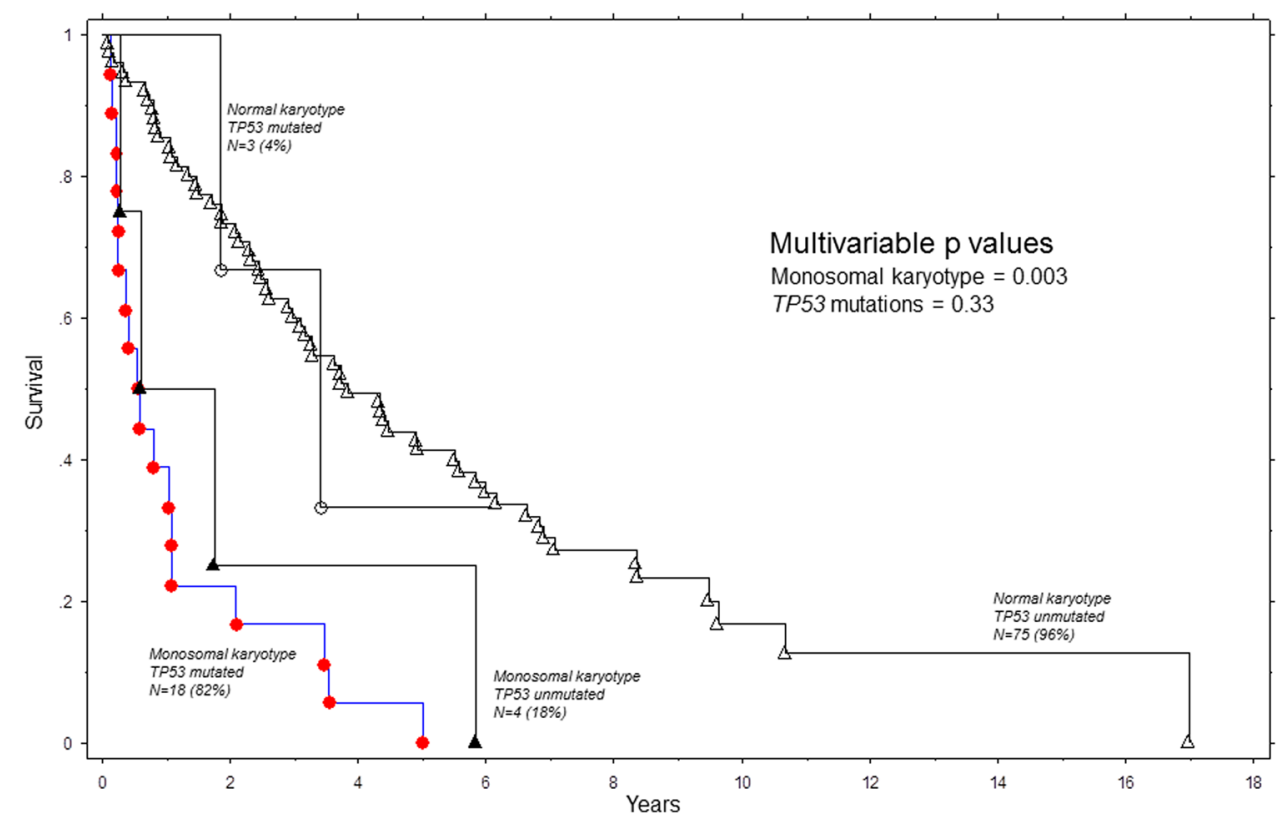

Fig. 1 Overall survival of 100 patients with primary myelodysplastic syndromes and either normal or monosomal karyotype, stratified by the presence or absence of TP53 mutations

affect its association pattern with trisomy 21; among the 19 cases with RUNX1 mutations, 13 involved the runt domain, 10 (53\%) were frameshift, 7 (37\%) missense, and 1 nonsense.

Given the adequate number of informative cases with monosomal karyotype $(n=22)$, we examined its prognostic interaction with TP53 mutations; in multivariable analysis that included these two variables, among 100 cases with either monosomal $(n=22)$ or normal $(n=78)$ karyotype, the prognostic contribution of TP53 mutations (HR 1.4; 95\% CI 0.7-3.0; $p=0.33$ ) was overridden by that of monosomal karyotype (HR 2.9; 95\% CI 1.4-5.9; $p=$ 0.003) (Fig. 1). Similar results were obtained when the entire cohort of 179 study patients were included in the multivariable model. Furthermore, in univariate analysis, monosomal karyotype $(p=0.04)$ and not TP53 mutations $(p=0.07)$ predicted leukemic transformation.

The observations from the current study carry both pathogenetic and prognostic relevance. The association between monosomal karyotype and TP53 mutations has also been recognized in the setting of acute myeloid leukemia $(\mathrm{AML})^{7}, 8$, and raises the possibility of mutationinduced genetic/chromosome instability as the cause of the particular cytogenetic abnormality. Furthermore, the current study suggests that the adverse impact of TP53 mutations might be accounted for by its association with monosomal karyotype, whose independent prognostic contribution in MDS has previously been stressed ${ }^{9,} 10$. The association between RUNX1 mutations and trisomy
21 was also previously reported in $\mathrm{AML}^{11}$, chronic myeloid leukemia, ${ }^{12}$ and B-cell acute lymphoblastic leuke$\mathrm{mia}^{13}$. Our observation on the association of SF3B1 mutations and $\operatorname{inv}(3)(q 21 \mathrm{q} 26.2)$ is novel and consistent with our previous observation of the same in chronic myelomonocytic leukemia ${ }^{14}$. As has also been previously noted in $\mathrm{MDS}^{15}$, SF3B1 mutations were also associated with del(11q), which is prognostically different from inv (3)(q21q26.2). These observations warrant careful attention to karyotype, when asserting the prognostic impact of mutation in MDS and other myeloid malignancies.

\section{Author details}

${ }^{1}$ Division of Hematology, Departments of Internal and Laboratory Medicine, Mayo Clinic, Rochester, MN, USA. ²Division of Hematopathology, Departments of Internal and Laboratory Medicine, Mayo Clinic, Rochester, MN, USA. ${ }^{3}$ Division of Cytogenetics, Departments of Internal and Laboratory Medicine, Mayo

Clinic, Rochester, MN, USA

\section{Competing interests}

The authors declare that they have no competing interests.

Received: 6 September 2017 Accepted: 13 September 2017

Published online: 18 December 2017

\section{References}

1. Bejar, R. et al. Clinical effect of point mutations in myelodysplastic syndromes. N. Engl. J. Med. 364, 2496-2506 (2011).

2. Tefferi, A. et al. Targeted next-generation sequencing in myelodysplastic syndromes and prognostic interaction between mutations and IPSS-R. Am. J. Hematol. 92, 1311-1317 (2017) 
3. Zhang, T. et al. High frequency of RUNX1 mutation in myelodysplastic syndrome patients with whole-arm translocation of $\operatorname{der}(1 ; 7)(q 10 ; p 10)$. Leukemia 31, 2257-2260 (2017).

4. Fernandez-Mercado, M. et al. Targeted re-sequencing analysis of 25 genes commonly mutated in myeloid disorders in del(5q) myelodysplastic syndromes. Haematologica 98, 1856-1864 (2013).

5. Vardiman, J.W. et al. The 2008 revision of the World Health Organization (WHO) classification of myeloid neoplasms and acute leukemia: rationale and important changes. Blood 114, 937-951 (2009).

6. Shaffer, L.G., Slovak, M.L., Campbell, L.J. (eds) ISCN 2009: An International System for Human Cytogenetic Nomenclature (2009): Recommendations of the International Standing Committee on Human Cytogenetic Nomenclature. (Karger Basel, 2009).

7. Rucker, F.G. et al. TP53 alterations in acute myeloid leukemia with complex karyotype correlate with specific copy number alterations, monosomal karyotype, and dismal outcome. Blood 119, 2114-2121 (2012).

8. Gaillard, J.B. et al. Monosomal karyotype routinely defines a poor prognosis subgroup in acute myeloid leukemia and is frequently associated with TP53 deletion. Leuk. Lymphoma 53, 336-337 (2012).

9. Patnaik, M.M. et al. Monosomal karyotype in myelodysplastic syndromes, with or without monosomy 7 or 5 , is prognostically worse than an otherwise complex karyotype. Leukemia 25, 266-270 (2011).
10. Gangat, N. et al. Evaluation of revised IPSS cytogenetic risk stratification and prognostic impact of monosomal karyotype in 783 patients with primary myelodysplastic syndromes. Am. J. Hematol. 88, 690-693 (2013).

11. Taketani, T. et al. AML1/RUNX1 mutations are infrequent, but related to AMLM0, acquired trisomy 21, and leukemic transformation in pediatric hematologic malignancies. Genes Chromosomes Cancer 38, 1-7 (2003).

12. Roche-Lestienne, $C$. et al. RUNX1 DNA-binding mutations and RUNX1PRDM16 cryptic fusions in BCR-ABL+leukemias are frequently associated with secondary trisomy 21 and may contribute to clonal evolution and imatinib resistance. Blood 111, 3735-3741 (2008)

13. Karrman, K. et al. High incidence of the ETV6/RUNX1 fusion gene in paediatric precursor B-cell acute lymphoblastic leukaemias with trisomy 21 as the sole cytogenetic change: a Nordic series of cases diagnosed 1989-2005. Br. J. Haematol. 135, 352-354 (2006)

14. Wassie, E.A. et al. Molecular and prognostic correlates of cytogenetic abnormalities in chronic myelomonocytic leukemia: a Mayo Clinic-French Consortium Study. Am. J. Hematol. 89, 1111-1115 (2014).

15. Stengel, A., Kern, W., Meggendorfer, M., Haferlach, T., \& Haferlach, C. MDS with deletions in the long arm of chromosome 11 are associated with a high frequency of SF3B1 mutations. Leukemia 2017. 